\title{
A dynamic model of interaction between reliance on automation and cooperation in multi-operator multi-automation situations
}

\author{
Ji Gao ${ }^{\mathrm{a}}$, John D. Lee ${ }^{\mathrm{a}, *}$, Yi Zhang ${ }^{\mathrm{b}}$ \\ ${ }^{a}$ Department of Mechanical and Industrial Engineering, The University of Iowa, 3131 Seamans Center, Iowa City, IA 52242, USA \\ ${ }^{\mathrm{b}}$ Department of Management Sciences, The University of Iowa, S210 John Pappajohn Business Building, Iowa City, IA 52242, USA
}

Available online 15 March 2006

\begin{abstract}
Flow management systems such as supply chain (SC) systems involve multiple operators managing elements of automation. The success of such multi-operator multi-automation systems depends not only on individual operator's appropriate use of automation, but also on cooperation between operators. Inappropriate reliance on automation may be a critical factor hindering cooperation and sharing automation-related information may improve reliance and promote cooperation. A computational model was developed to investigate how individual operator's use of automation influences cooperation between individuals. The model consists of an extension of the decision field theory [Busemeyer, J.R., Townsend, J.T., 1993. Decision field theory: a dynamic cognitive approach to decision making in an uncertain environment. Psychological Review 100(3), 432-459] that simulates the dynamics of an individual's trust and reliance on automation and a game theoretic framework that simulates the dynamics of cooperation between individuals in an iterated Prisoners' Dilemma game. Simulation experiments with this model were conducted to test the following hypotheses in the context of a twomanufacturer one-retailer SC system: (1) inappropriate use of automation will hinder cooperation; (2) sharing information regarding the performance of automation will directly improve reliance on automation and reduce unintentional competitive behavior; and (3) sharing information regarding the reliance on automation will lead to a more charitable interpretation of the other's intent to cooperate or compete and therefore promote cooperation. The simulation results show that inappropriate reliance on automation undermines cooperation and sharing information of automation performance and reliance improves cooperation. The degree of improvement in cooperation by sharing such information is greatest in situations in which automation recovers from faults.
\end{abstract}

\section{Relevance to industry}

Promoting cooperation is important for decentralized systems such as SCs where inappropriate use of automation may undermine cooperation. This paper shows how reliance on automation influences cooperation and how sharing automation-related information mediates this influence. The results suggest some ways to promote sustainable cooperation.

(C) 2006 Elsevier B.V. All rights reserved.

Keywords: Trust; Automation; Horizontal cooperation; Information sharing; Game theory; Supply chain

\section{Introduction}

Reliance on automation describes the process of an operator's using or engaging automation (Lee and See, 2004). Inappropriate reliance on automation has contributed to numerous industrial disasters and such disasters

\footnotetext{
*Corresponding author. Tel.: + 13193840810.

E-mail addresses: jgao@engineering.uiowa.edu (J. Gao), jdlee@engineering.uiowa.edu (J.D. Lee), yi-zhang-2@uiowa.edu (Y. Zhang).
}

may become increasingly common and catastrophic as automation becomes more prevalent (Parasuraman and Riley, 1997). Most previous research has focused on how a single operator manages one or a few elements of automation. However, many systems include multiple operators managing multiple elements of automation. We call these systems multi-operator multi-automation (MOMA) systems. The successful operation of such MOMA systems depends not only on operators' appropriate reliance on the automation, but also on the cooperation between operators. In the present study we 
define cooperation as 'joint action for mutual benefit' (Dugatkin et al., 1992). In addition to directly affecting system performance, operators' inappropriate reliance on automation may undermine their cooperation with other operators.

Lack of cooperation, between operators in a MOMA system such as a power grid, can cause severe problems. As an example, the worst power grid failure in US history occurred on August 14, 2003. The flow of approximately $61,800 \mathrm{MW}$ of electricity was disrupted, leaving 50 million customers from Ohio to New York and parts of Canada without electric power (Lipton et al., 2003). An important contributor to this event was a lack of cooperation between two regional electrical grid operators who were monitoring the same region (US-Canada Power System Outage Task Force, 2003). These operators managed the flow of electricity from suppliers to distributors. A failure to exchange information about their operations prevented them from understanding and responding to changes in the power grid.

Cooperation between network members is also critical for supply chain (SC) systems. A SC is a network of interlinked suppliers, manufacturers, distributors, and retailers. Effective SC management (SCM) requires a high degree of collaboration between supply chain members (Wu and O'Grady, 2004). The advantages of cooperation between upstream and downstream SC members, referred to as vertical cooperation, have been widely acknowledged (Cachon and Zipkin, 1999; Sahin and Robinson, 2002). However, the value of cooperation between substitutable or competing SC members, referred to as horizontal cooperation, has not been fully recognized. There is an increasing need for horizontal cooperation in SCs. For example, with soft-drink products, cooperation and the sharing of information between various local distributors of the same brand in one geographical area generally benefit all. In the automotive parts industry, developing more horizontally cooperative relationships with those who are typically competitors has become a major trend (Burgers et al., 1993; Dussauge et al., 2004). In the European print industry, cooperating with competitors results in better service to customers. Horizontal cooperation between farm businesses has been shown to help them acquire more useful information, make more effective use of new technologies, and improve purchasing power (Napier, 2001).

Although cooperation has been recognized as an important factor in the success of a SC system, cooperation has proved difficult to sustain (Sabath and Fontanella, 2002). Effective cooperation is built on a foundation of trust and lack of trust can undermine cooperation (Barratt, 2004; Lee and Billington, 1992). The advantage of cooperation can only be realized if the cooperation is sustained over time. Short-term opportunistic behavior can undermine future cooperation by increasing the level of distrust between participants (Kumar and vanDissel, 1996). Therefore, there is a need to understand what conditions enhance trust between operators and promote cooperation. Little attention has been given to the unintentional competitive behavior and the misinterpretation of others' intent that can induce competitive behavior. Inappropriate use of automation may cause such unintentional competitive behavior and generate the misinterpretation that initiates increasingly competitive behavior. In this study, we investigate how to reduce such competition and promote cooperation by improving appropriate reliance on automation with the sharing of automationrelated information.

Both power grid and SCM are examples of the general domain of flow management, in which MOMA performance is particularly important. A linked structure of multiple flows and reservoirs defines a decentralized network managed by multiple operators with the support of multiple elements of automation. Very few studies have addressed how the interaction between multiple operators and multiple automation elements influences cooperation in decentralized systems. To the best of the authors' knowledge, no research has investigated how an individual's reliance on automation influences the cooperation between individuals in the context of a SC system, which is the focus of this study.

The paper is organized as follows: first the SC scenario and the game situation considered are described; then the rationale for sharing automation-related information is presented; a model of interaction between reliance on automation and cooperation is introduced; simulation experiments with the model are described; and finally results showing how different types of information sharing influence cooperation are presented.

\section{Supply chains and game situations}

It is well recognized that cooperation between SC members can increase system-wide efficiency (Cachon, 2002). A review of related articles shows that cooperation in SC systems can be categorized into two types: vertical and horizontal cooperation (Anupindi et al., 2001). Vertical cooperation refers to the cooperation between upstream and downstream SC members (e.g., between supplier and manufacturer or manufacturer and retailer). For example, there might be conflicting interests between the manufacturer and the retailer: the retailer prefers a high frequency of small orders to avoid inventory costs while the manufacturer prefers a low frequency of larger orders to benefit from batch production. Vertical cooperation between the manufacturer and the retailer might produce a medium-order frequency and a medium-order size to accommodate both. Competition can only emerge when each member considers their own constraints without a willingness to compromise. Horizontal cooperation refers to the cooperation between SC members who play the same role at the same stage of a SC such as the potential entrants, substitutes, and competitors (e.g., between multiple suppliers, multiple manufacturers, or multiple retailers) 
(Anupindi et al., 2001). The horizontal SC structure often creates a game situation between SC members in that the members share the same market, where the interests are both consistent and conflicting (Luce and Raiffa, 1957; von Neumann and Morgenstern, 1944). They have conflicting interests because each of them wants a larger share of the demand or market than the other.

In a SC where two manufacturers supply one retailer, a higher level of inventory is needed by one manufacturer to get a larger market share than the other manufacturer. They have consistent interests in that they both want to avoid unnecessarily high inventory associated with high holding costs. Therefore, on one hand, both want to keep a high level of inventory to be able to meet a large proportion of the demand; on the other hand, both want to keep a low level of inventory to avoid high inventory costs. This type of inventory-based competition between multiple firms who provide substitutable products or competing brands of the same product has been well established (Lippman and McCardle, 1997; Mahajan and van Ryzin, 2001; Parlar, 1988). An early treatment of the substitutable product inventory problem is given by Parlar (1988) in a two-retailer game situation. More recent research has investigated multiple retailers competing for a single good supplied by one manufacturer (Anupindi et al., 2001; Lippman and McCardle, 1997; Rudi and Netessine, 1999). Other studies have examined the horizontal interaction between multiple manufacturers sharing one retailer or between multiple suppliers sharing one manufacturer (Elahi et al., 2003; van Ryzin and Mahajan, 1999). All these studies describe the strategic relationship between multiple horizontal SC members in a game situation in which, although cooperation might benefit all SC members, all members might also serve their own interests best by competing. In this paper, we consider horizontal cooperation between two manufacturers in a two-manufacturer one-retailer SC system where two manufacturers share the same market.

How the responsibility for managing inventory is allocated between the manufacturer and the retailer has a strong influence on cooperation. Generally, there are two approaches: traditional Retailer-Managed Inventory (RMI) and Vendor-Managed Inventory (VMI). RMI refers to situations where the retailer monitors and manages the inventory, while VMI applies to situations where the retailer delegates its inventory management to its upstream manufacturer or supplier. A recent study investigated the reasons why retailers prefer VMI to traditional RMI from the perspective of competition between manufacturers of competing brands (Mishra and Raghunathan, 2004). A situation where a single retailer sells two brands of a substitutable product provided by two manufacturers was considered. The analysis showed that VMI intensifies the competition between manufacturers of competing brands compared to RMI. The increased competition under VMI causes manufacturers to stock more than what the retailer would have stocked under RMI. VMI rather than RMI is used in the present study because it captures an increasingly common SC characteristic that induces inventorybased competition between manufacturers in a twomanufacturer one-retailer SC.

With the inventory management strategy of VMI, how the demand is allocated among manufacturers defines the game situation. Lippman and McCardle (1997) analyzed a competitive newsboy (oligopoly) model. They analyzed the situation where aggregate demand is allocated to firms based on splitting rules. A splitting rule specifies how initial demand is allocated among competing firms before the demand is realized and how any excess demand is allocated among firms with remaining inventory after the demand is realized. They examined the relationship between equilibrium inventory levels of competing firms and the demand splitting rules and provided conditions under which there is a unique equilibrium. Another study also considered the inventory-based competition between multiple suppliers sharing demand and the demand is split according to an allocation rule (Elahi et al., 2003). This demand-splitting rule that allocates demand to each supplier based on the amount of inventory each holds is referred to as a stockproportional scheme. This scheme defines a symmetric game situation where both players have incentives to compete, but competition turns out to be inferior to both cooperating.

In this study, the horizontal interaction between manufacturers in a two-manufacturer one-retailer SC system is considered. VMI management and the stockproportional demand allocation rule are used to generate an inventory-based game between two manufacturers. Multiple manufacturers sharing the same market is a very common SC structure, characterizing a more general multisource SC that has great potential to improve system efficiency and service compared to a single-source SC. This structure provides a realistic and yet relatively simple context to examine how individual's reliance on automation and sharing automation-related information may influence the dynamics of cooperation between SC members.

\section{Information sharing and types of automation in SC}

Information sharing is an important factor that influences the level of cooperation in SCM. Information sharing generally describes situations where SC partners exchange SCM-related data such as current order and production status as well as plans and forecasts. Many studies have compared situations with information sharing to those with no or limited sharing (Gavirneni et al., 1999; Huang et al., 2003; Lee and Whang, 2000; Sahin and Robinson, 2002). The general finding is that information sharing is beneficial to all SC members especially the upstream ones. A few studies have explored the effect of information sharing on horizontal cooperation in SCs $(\mathrm{Li}, 2002$; Raghunathan, 2003; Zhang, 2002). However, these studies considered the horizontal cooperation between two SCs 
and the vertical information sharing between upstream and downstream SC members within each SC. How horizontal information sharing influences the horizontal cooperation in itself merits attention. Also, most studies consider only information such as inventory, production schedule, orders, sales, or forecasts. Little research has explored the role of sharing automation-related information in SCs. This study investigates how sharing automation-related information influences cooperation.

Automation can be categorized into four types according to the nature of its function: information acquisition, information analysis, decision and action selection, and action implementation (Parasuraman et al., 2000). These categories also apply to SC automation. Automation of information acquisition senses and registers input data. An example is Enterprise Resource Planning (ERP) software. One function provided by ERP is to collect input data from various SC members and then make the information accessible to all. Automation of information analysis involves data inference and prediction. A forecasting system that records history demand and applies a model to make a demand forecast would be an example of such automation. Automation of decision and action selection involves hypotheses generation and selection from among decision alternatives. Decision support systems such as advanced planning and scheduling (APS) are examples that can produce detailed production schedules and make inventory planning decisions based on input data. Automation of action implementation refers to the actual execution of the action. Some widely used action automation in SCs includes manufacturing execution systems, transportation execution, and warehouse management systems. In practice, automated systems in SC generally encompass more than one function. For instance, ERP not only collects input data but also makes decisions for inventory planning.

As automation becomes more prevalent in SCM, the performance of SC operations will depend more on whether decision makers rely appropriately on automation. For example, how appropriately the decision maker relies on the forecasting decision support system to make a demand forecast has critical implications for other decisions such as production planning. The present study proposes that inappropriate reliance on automation may influence SC performance in two ways. First, inappropriate reliance on automation can directly affect the SC performance in that use of automation significantly influences SC success. Second, inappropriate reliance can lead to unintentional competitive behavior, which undermines cooperation and consequently the SC performance. Two types of automation-related information may improve SC performance: information regarding the performance of the automation and information regarding reliance on automation. Automation performance information reflects the capability of the automation to achieve the operators' goals. The information regarding automation performance of other SC members may help the decision maker assess the capability of the automation and therefore rely on automation more appropriately. As a consequence, an unintentional competitive behavior is less likely to occur due to improved reliance on automation. The information regarding the other's reliance on automation may help the decision maker more precisely interpret the other's intent to cooperate or compete regardless of any specific behavior. In this study, we will examine how sharing information regarding the performance of automation and information regarding the reliance on automation influences decision makers' reliance on automation as well as the dynamics of cooperation with the simulation experiments.

\section{A model of multi-operator multi-automation (MOMA)}

A complex SC network can be regarded as a MOMA system where multiple decision makers use multiple elements of automation to manage the SC. An integrated model of a MOMA system is developed to describe the interaction between operators and automation as well as the cooperation among multiple operators. The model includes extended decision field theory (EDFT) that describes the dynamics of an individual operator's trust and reliance on automation and a game theoretic framework that describes the dynamics of cooperation between operators. These components are integrated to describe how reliance on automation influences cooperation and how sharing different types of automation-related information influence cooperation.

The model will be described in the context of the twomanufacturer one-retailer SC introduced in Section 2. The task for the decision maker is to first set the target inventory level of the retailer and then to make a production planning decision based on the target inventory and the demand forecast periodically (e.g., monthly). The production planning decision consequently determines the actual inventory level at the retailer. When the actual demand is realized, the payoff is determined based on the actual inventory levels of both manufacturers. There are generally many elements of automation involved in this process and only one, the forecasting system, is considered in this study. It is assumed that the two manufacturers use a similar forecasting system. In the following context, the automation refers to the forecasting system and operators refer to decision makers in each manufacturing unit of the SC. Reliance on automation refers to whether the operator uses the forecast suggested by the forecasting system (automation) or his/her own judgmental forecast (manual control). The appropriateness of use of automation refers to whether the decision maker uses the forecast suggested by the forecasting system when it is accurate and does not use it when it is inaccurate.

\subsection{Extended decision field theory (EDFT)}

DFT provides a rigorous mathematical framework to describe the deliberation process involved in making 
decisions under uncertainty (Busemeyer and Townsend, 1993). DFT differs from most decision-making approaches by being stochastic and dynamic rather than deterministic and static (Townsend and Busemeyer, 1995). However, DFT only considers the time course of cognition (e.g., preference for alternative choices) preceding a single decision, not the time course of multiple sequential decisions in dynamic situations where sequential decisions are interdependent. That is, DFT does not consider the effect of previous decisions on the next decision in multiple sequential decisions. Therefore, DFT was extended to consider the multiple sequential decisions inherent in a MOMA context (Gao and Lee, in press).

The focus of consideration here is the individual operator who makes sequential decisions about whether to rely on the automation (A) or use manual control (M). The EDFT links the sequential decisions by dynamically updating the operator's belief in the automation $\left(B_{\mathrm{A}}\right)$ or manual control capability $\left(B_{\mathrm{M}}\right)$ based on previous experiences, which guides the next decision. The operator's belief of automation, $B_{\mathrm{A}}$, is updated as (Gao and Lee, in press)

$B_{\mathrm{A}}(n)=\left\{\begin{array}{l}B_{\mathrm{A}}(n-1)+\frac{1}{b}\left(C P_{\mathrm{A}}(n-1)-B_{\mathrm{A}}(n-1)\right), \\ \quad \text { if } C P_{\mathrm{A}}(n-1) \text { is available, } \\ B_{\mathrm{A}}(n-1), \quad \text { otherwise, }\end{array}\right.$

where $b$ represents the level of transparency of the system interface, describing how well the available information is conveyed through the interface to the operator. The value of $b$ is equal to 1 (perfectly conveyed) or greater than 1 (poorly conveyed). $C P_{\mathrm{A}}$ denotes the automation capability. The same formula applies to update the operators' belief in manual control capability, $B_{\mathrm{M}}$, by replacing $B_{\mathrm{A}}$ with $B_{\mathrm{M}}$ and $C P_{\mathrm{A}}$ with $C P_{\mathrm{M}}$ where $C P_{\mathrm{M}}$ denotes the manual control capability. The capability of a control (either A or $\mathrm{M})$ is defined as the degree to which the control accomplishes the target performance set by the user. This capability varies over time. It is assumed that the performance of a control is exclusively determined by the capability of that particular control. Eq. (1) updates the operator's belief in automation or manual control capability using the previous performance of the control if this performance information is available, and without any update if the information is unavailable.

One of the key concepts in DFT is that the preference for alternative choices evolves over time based on the accumulated information (Busemeyer and Townsend,
1993). A similar formula to the updating formula of preference in DFT is applied to trust in automation $(T)$ and self-confidence $(S C)$ in EDFT (Gao and Lee, in press):

$T(n)=(1-s) T(n-1)+s B_{\mathrm{A}}(n)+\varepsilon(n)$,

$S C(n)=(1-s) S C(n-1)+s B_{\mathrm{M}}(n)+\varepsilon(n)$.

The parameter, $s$, is the growth-decay rate, representing how strongly the current state depends on the past state. The noise term $\varepsilon$ has normal distribution $N\left(0, \sigma^{2}\right)$, representing the uncertainty of trust or self-confidence. The initial values of $T$ and $S C, T(0)$ and $S C(0)$, are assumed to be equal and are referred to as $z$. The preference $(P R)$ for $\mathrm{A}$ over $\mathrm{M}$ is defined as the difference between trust in automation and self-confidence (Gao and Lee, in press):

$P R(n)=T(n)-S C(n)$.

The model predicts that, for the period $n$, the operator will choose to rely on automation when $P R(n)$ evolves beyond $\theta^{+}$, to use manual control when $P R(n)$ evolves below $\theta^{-}\left(=-\theta^{+}\right)$, and repeat the previous choice when $\operatorname{PR}(n)$ is between $\theta^{-}$and $\theta^{+}$. The inhibitory threshold, $\theta^{+}$ (or $-\theta^{+}$), defines the minimum difference between trust and self-confidence needed to transition from automation to manual control or vice versa. Table 1 summarizes the meaning of each parameter and the values used in this study.

The inputs of the EDFT model are $C P_{\mathrm{A}}$ and $C P_{\mathrm{M}}$ and the output is the operator's decision to use automation or manual control, that is, the reliance on automation. By comparing the reliance on automation with the corresponding values of $C P_{\mathrm{A}}$ and $C P_{\mathrm{M}}$, the appropriateness of the use of the automation can be derived. Reliance on automation when $C P_{\mathrm{M}}$ is greater than $C P_{\mathrm{A}}$ is inappropriate.

This dynamic model of trust, self-confidence, and reliance replicates several empirical phenomena including the tendency of operators to adopt an all-or-none reliance strategy and the inertia of trust and reliance (Gao and Lee, in press). EDFT provides a well-defined computational structure to operationalize the conceptual model of trust, self-confidence, and reliance on automation (Lee and See, 2004). Fig. 1 shows how this model describes the dynamic closed-loop relationship between the context and the operator's decision to rely on automation.

Table 1

Summary of parameters for EDFT model

\begin{tabular}{lll}
\hline Parameter & Meaning & Value \\
\hline$b$ & Level of transparency of the system interface, accounting for how much the latest experience affects the belief & 1 \\
$z$ & Initial value of trust (or self-confidence) & $\left(C P_{\mathrm{A}}(1)+C P_{\mathrm{M}}(1)\right) / 2$ \\
$s$ & Growth-decay rate, representing how strongly the current state depends on the past state & 0.8 \\
$\sigma^{2}$ & Variance of the noise term, $\varepsilon$, representing the uncertainty of trust (or self-confidence) & 0.05 \\
$\theta$ & Decision threshold, accounting for speed-accuracy tradeoff effect & $2 \sigma$ \\
\hline
\end{tabular}




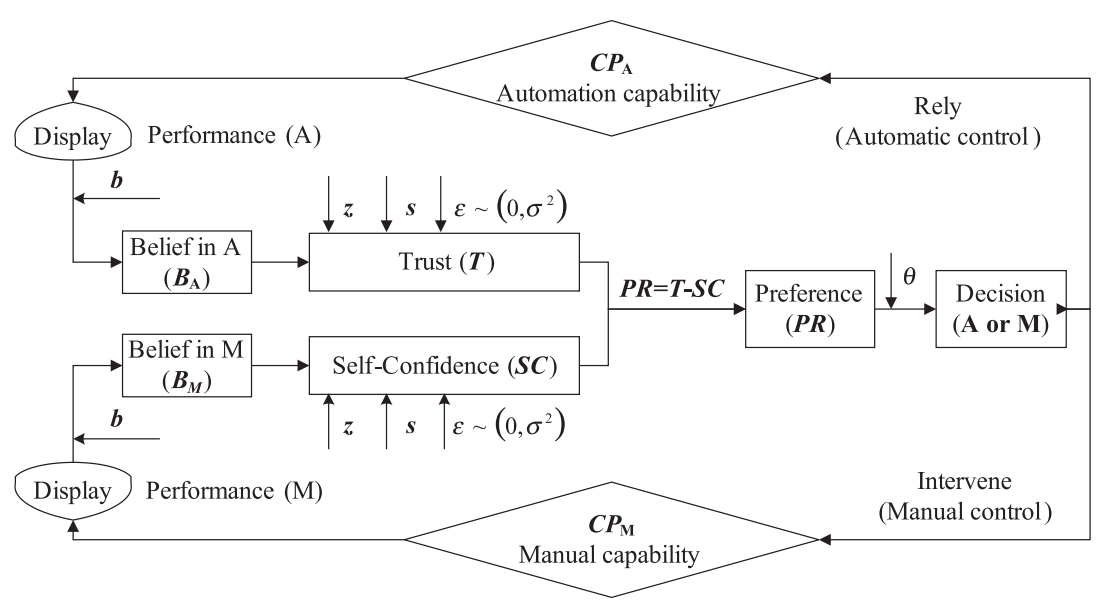

Fig. 1. Conceptual model of EDFT for operators' reliance on automation.

\subsection{Game theoretic framework}

A critical element of flow management concerns the cooperative or competitive strategies adopted by the operators. Game Theory (GT) provides a useful formalism to investigate the dynamics of cooperative relationships (von Neumann and Morgenstern, 1944). GT has become an essential tool in the analysis of SCM systems in which multiple agents have conflicting objectives (Narasimhan and Mahapatra, 2004). A recent review of GT applications in SCM surveys the types of games explored by these studies (Cachon and Netessine, 2004). Games can be categorized based on different attributes. For instance, games can be classified into strictly cooperative, strictly competitive, or mixed motive games. The players' preferences among the outcomes are identical in strictly cooperative games (i.e., pure coordination games), diametrically opposed in strictly competitive games (i.e., zerosum games), and neither identical nor diametrically opposed in mixed motive game (i.e., non-zero-sum games) (Colman, 1982). Games can also be categorized into static or dynamic games according to whether they are 'one-shot' or iterated games. A game can also have complete or incomplete information depending on whether the set of strategies and payoffs are known to each player. The concept of complete or incomplete information can be applied to any game, but the concept of perfect or imperfect information is applied only to iterated games. An iterated game can be considered to have perfect or imperfect information depending on whether a player knows exactly what decisions other players have made up to that point. Most applications of GT to SCs have focused on static games ('one-shot' games) as well as on games with complete and perfect information (Cachon and Netessine, 2004).

The two-manufacturer one-retailer SC system described in Section 2 generates a mixed motive game between manufacturers (i.e., one with both common and conflicting interests). It is a dynamic game situation (i.e., iterated)
Table 2

Payoff matrix expected by both manufacturers (forecasting $=100$ units)

\begin{tabular}{llll}
\hline & & Manufacturer 2 & \\
\cline { 3 - 4 } & & Cooperate $(C)$ & Compete $(D)$ \\
\hline Manufacturer 1 & Cooperate $(C)$ & $R, R(50,50)$ & $S, T(38,57)$ \\
& Compete $(D)$ & $T, S(57,38)$ & $P, P(45,45)$ \\
\hline
\end{tabular}

$R$ : reward; $P$ : punishment; $T$ : temptation; $S$ : sucker, standard terms used in PD

because the two manufacturers decide to compete or cooperate over multiple periods. More specifically, it is a time-dependent iterated game because many variables in SCs, such as demand, vary over time. Due to time dependency, given the same set of choices by two manufacturers regarding whether to cooperate or compete, the set of payoffs calculated based on the combined choices will be different in different periods.

A payoff matrix in GT shows what payoff each player will receive, depending on the combined choices of all players. In a game of two players, two choices, cooperate (C) or defect $(D)$, intersect to create four outcomes, each with a designated payoff: $R$ denotes the reward for mutual cooperation, $P$ the punishment for mutual defection, $T$ the temptation to defect, and $S$ the sucker's payoff. It is defined that, when $T>R>P>S$ and $2 R>T+S$, the game is a Prisoners' Dilemma (PD) game, a well-known mixedmotive game in which two prisoners can either compete and confess or cooperate and not confess (Davis, 1983). Table 2 shows an example of the payoff matrix of a PD game. The left number in each cell represents the payoff for player 1 and the right number represents the payoff for player 2 for each of four outcomes. In conventional GT, a player is faced with a set of choices he can make and will choose a best response to other players' actions. When the best responses of all players are in accordance with each other, a so-called 'Nash Equilibrium' will be reached 
(Nash, 1950). With a payoff matrix such as the one shown in Table 2, when playing only one game, it is clear that the best response to each player is to compete regardless of the opponent's choice. However, when both players choose to compete, the outcome is worse for both players, called Pareto-inferior (to mutual cooperation) in GT. Thus the type of game situation demonstrated in Table 2 represents a PD game where both players competing constitutes the Nash equilibrium, from which neither player has incentive to change unilaterally.

In terms of the payoff matrix, the time-dependent iterated game considered in the present study has different payoff matrices in different periods. Furthermore, different manufacturers might have different payoff matrices in the same period. For example, different demand forecasts will generate different payoff matrices. When the payoff matrix is not known to both manufacturers, the game becomes one of incomplete information. Each manufacturer can only infer the other's choice to cooperate or to compete based on their interpretation of the other's previous behavior, which might not fully reflect the other's true intent. Therefore, this situation is an iterated game of imperfect information.

In this simulation study, the game between two manufacturers becomes a PD game with the following characteristics. First, the manufacturers are free to set their target inventory level and incur inventory holding costs individually. Because the inventory located at the retailer directly satisfies customer demand, the manufacturer who holds more inventory than the other shares a larger proportion of the demand or market. Therefore, each manufacturer's target inventory level indicates his intent to cooperate or compete. To represent this SCM behavior as a PD game, setting the target inventory level at $50 \%$ or less of the expected demand represents cooperation $(C)$ and setting the target inventory level at $75 \%$ or above of the expected demand represents competition with intent to defect $(D)$. Seventy-five percent was chosen to define competition so that the payoff of the cooperation or competition could be clearly distinguished. Second, the cost parameters also influence the game structure. The profit earned per unit product sold $(r)$ and the inventory cost per unit product per time period $(h)$ are fixed: $r=1$ and $h=0.2 r$. The ratio of the inventory cost to the unit profit determines the nature of the game. Simulation results based on the proposed model show that the game is a PD game only when the ratio is between 0 and 0.65 , and so $50 \%$ and $75 \%$ of the expected demand are used to define cooperation and competition. The external demand is given as a constant of 100 units. Table 2 shows the expected payoff matrix for the period when both manufacturers have accurate demand forecasts (other factors influencing the inventory level are not considered). Although the expected payoff matrix, which defines the incentive to cooperate and compete, might be different for different periods because of different demand forecasts, the basic structure of the payoff matrix remains that of a PD game.

\subsection{A model of cooperation dynamics}

An iterated Prisoners' Dilemma (IPD) is the most frequently used framework for studying the evolution of cooperation among selfish agents when there is a shortterm temptation to cheat (Dawkins, 1988; Sigmund, 1993; Smith, 1982). GT approaches have been used to describe the dynamics of cooperation in the IPD games. As introduced previously, conventional GT assumes that players always choose a best response to other players' actions to maximize their individual expected payoffs. That is, players' forward-looking rationality is one of the most important assumptions of GT. However, choices that should be made according to GT have little resemblance to actual decision making (Macy and Flache, 2002). Experimental evidence has shown that GT cannot explain the data because human decision makers do not follow its rational prescriptions (Colman, 1995; Foddy et al., 1999; Ledyard, 1995; Simon, 1992). Assumptions of rationality become particularly problematic in games in which individuals have only partial control over the outcomes because expected payoff maximization is undefined in the absence of assumptions about how the other participants will behave (Colman, 2003). In addition, the Nash equilibrium, the main solution concept in conventional GT, provides indeterminate choice between equilibriums when a game has multiple equilibriums and therefore cannot describe the dynamics by which a population of players moves from one equilibrium to another (Colman, 2003; Macy and Flache, 2002).

These limitations of conventional GT, along with concerns about the cognitive demands of forward-looking rationality, have motivated efforts to explore backward-looking alternatives to understand human decision making in a game situation. Learning-based describes human decision-making behavior in iterated game situations (Colman, 2003; Gutnisky and Zanutto, 2004; Macy, 1991; Macy and Flache, 2002). Conventional GT adopts an analytical approach, which assumes that players have sufficient cognitive capacity to make accurate predictions about the payoffs of the different decisions; learning theory makes assumptions more consistent with the cognitive capacity of the agents by assuming that they base these predictions on experience and induction rather than logic and deduction (Macy and Flache, 2002). A substantial proportion of behavior observed in experimental IPD games has been cooperative, which cannot be predicted by strict rationality in conventional GT (Andreoni and Miller, 1993; Cooper et al., 1996; Selten and Stoecker, 1986). For example, an equilibrium of mutual cooperation is typically observed. Experimental evidence has shown that learning might contribute to greater cooperation and to equilibrium of mutual cooperation in IPD games (Lew et al., 2001; Stephens et al., 2002). On the basis of this evidence, a learning-based approach is chosen to model the dynamics of cooperation.

Reinforcement learning describes decision making in IPD, where behavior adapts to payoffs over time. Rewards 
associated with a choice generate a tendency to repeat that choice (even if other choices might have higher rewards); punishments associated with a choice generate a tendency to avoid that choice and switch to alternative choices (even if alternative choices have the potential to be even worse). Macy and Flache (2002) applied reinforcement learning to a game situation, in which the payoff associated with a choice was evaluated as satisfactory or unsatisfactory relative to an aspiration level, $A L$. The aspiration level, $A L$, can be interpreted as the expected payoff when behavioral propensities are uninformed by prior experience, such that the possibility of each of four payoffs is equal. Whether the payoff is satisfactory or unsatisfactory is indicated by the stimulus $s_{\mathrm{a}, t}(a \in\{C, D\})$, which is calculated as (Macy and Flache, 2002)

$s_{\mathrm{a}, t}=\frac{\pi_{\mathrm{a}, t}-A L}{\max [|T-A L|,|R-A L|,|P-A L|,|S-A L|]}$,

$a \in\{C, D\}$,

where $\pi_{\mathrm{a}, t}$ is the actual payoff associated with choice $a$ for period $t$. Considering an IPD game with the payoff matrix as shown in Table 2 where the four possible payoffs are $R$, $P, T$, and $S$, a simple and reasonable estimate of the aspiration level is the average of the four payoffs (Macy and Flache, 2002):

$A L=(T+R+P+S) / 4$.

$s_{\mathrm{a}, t}\left(-1 \leqslant s_{\mathrm{a}, t} \leqslant 1\right)$ represents a positive or negative stimulus, corresponding to rewards or punishments associated with the choice taken. The probability of taking the associated choice is updated based on the rewards and punishments experienced (Macy and Flache, 2002).

\subsubsection{Extension of reinforcement learning theory}

The choice determined by the probability in the reinforcement learning theory produces an oscillation between cooperation and competition that slowly reaches mutual cooperation (Macy and Flache, 2002). In the present study, we use a similar concept of preference and threshold from EDFT described in Section 4.1 to determine the player's choice to cooperate or compete. This produces less variable cooperation dynamics, which is more consistent with the empirical results.

Similar to how the preference and a threshold determine whether a decision is made in EDFT, the decision of selecting a particular choice ( $C$ or $D$ ) is determined by the evolving preference and a threshold in the present study. The player's preference of cooperation $(C)$ over competition $(D)$ for period $t+1, P R_{C / D, t+1}$, is calculated as

$$
\begin{aligned}
& P R_{C / D, t+1} \\
& \quad= \begin{cases}(1-l) P R_{C / D, t}+l s_{a, t} & \text { if } a_{t}=C, \\
(1-l) P R_{C / D, t}-l s_{a, t}, & \text { if } a_{t}=D,\end{cases}
\end{aligned}
$$

where $a \in\{C, D\}$ where $P R_{C / D, t}$ is the preference at the previous period, $t$, and $l$ is the learning rate $(0<l<1)$. The preference at the first period, $P R_{C / D, 1}$, represents the player's initial tendency to cooperate or to compete, without experiencing any payoffs from choices. An intermediate value of learning rate, $l=0.5$, is selected because it has been widely used (Macy and Flache, 2002). An unbiased initial preference, $P R_{C / D, 1}=0$, is also used. The updating rule of the preference shows that exposure to a positive stimulus when selecting $C$ or exposure to a negative stimulus when selecting $D$ will increase the preference of $C$ over $D$, otherwise, it will decrease the preference.

The preference is updated over time for the iterated game. The player will choose $C$ over $D$ when the preference evolves beyond a threshold, $\theta_{C / D}^{+}$(upper threshold), will choose $D$ over $C$ when the preference evolves below $\theta_{C / D}^{-}$ (lower threshold), and will repeat the previous choice when the preference is between $\theta_{C / D}^{-}$and $\theta_{C / D}^{+}$. The level of the threshold can reflect a player's willingness to cooperate or to compete. A lower level of $\theta_{C / D}^{+}$or $\theta_{C / D}^{-}$will be used to represent the situation where people are more willing to cooperate compared with a higher level of $\theta_{C / D}^{+}$or $\theta_{C / D}^{-}$. For each period, the model's output is a prediction of an operator's choice regarding the decision to cooperate or compete as the output. Fig. 2 summarizes the dynamic closed-loop relationship between each operator's decision to cooperate or compete and the actual behavior of both operators.

\subsection{Integrated model}

Fig. 3 shows the integrated model of a MOMA system, which consists of a model of EDFT and a game theoretic model of cooperation dynamics. The two models are integrated in such a way that the output of the EDFT model is also the input of the cooperation model. This integrated model will be described in the context of a twomanufacturer one-retailer SC system using the automation of forecasting systems.

As shown in Fig. 3, the model of EDFT illustrates how an individual operator relies on automation. Trust in automation guides reliance on automation and therefore influences the associated task performance. The performance will then influence the trust and reliance in the next period in a closed-loop fashion. Inappropriate use of the forecasting system will generate inaccurate forecasts. When the actual demand is realized and the forecasting performance is observed, operators can adjust their trust and reliance on the forecasting system for the next period.

The output of the EDFT model, reliance on automation, becomes the input to the cooperation model. The operators' intent to cooperate or to compete is defined by the target inventory level, which can be described in terms of the percentage of the forecasted demand (i.e., $50 \%$ or $75 \%$ ). However, the actual inventory level, which can be described in terms of the percentage of the actual demand, might deviate from the target level. The magnitude of the 


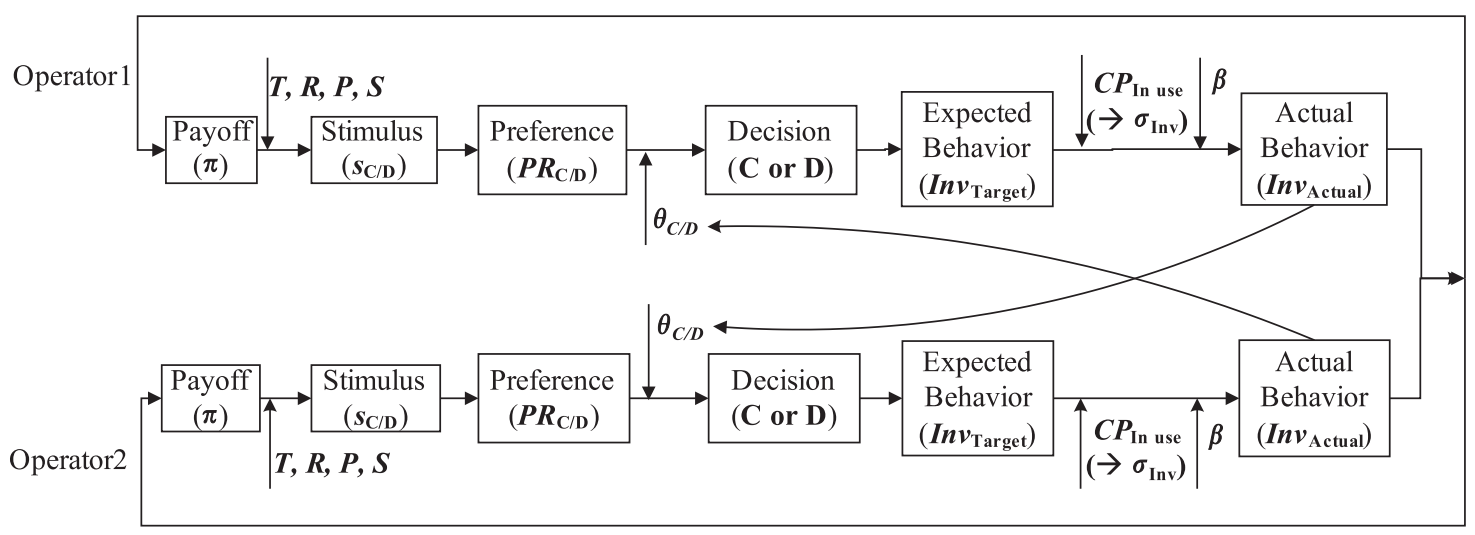

Fig. 2. Conceptual model of cooperation dynamics for two players (operators).

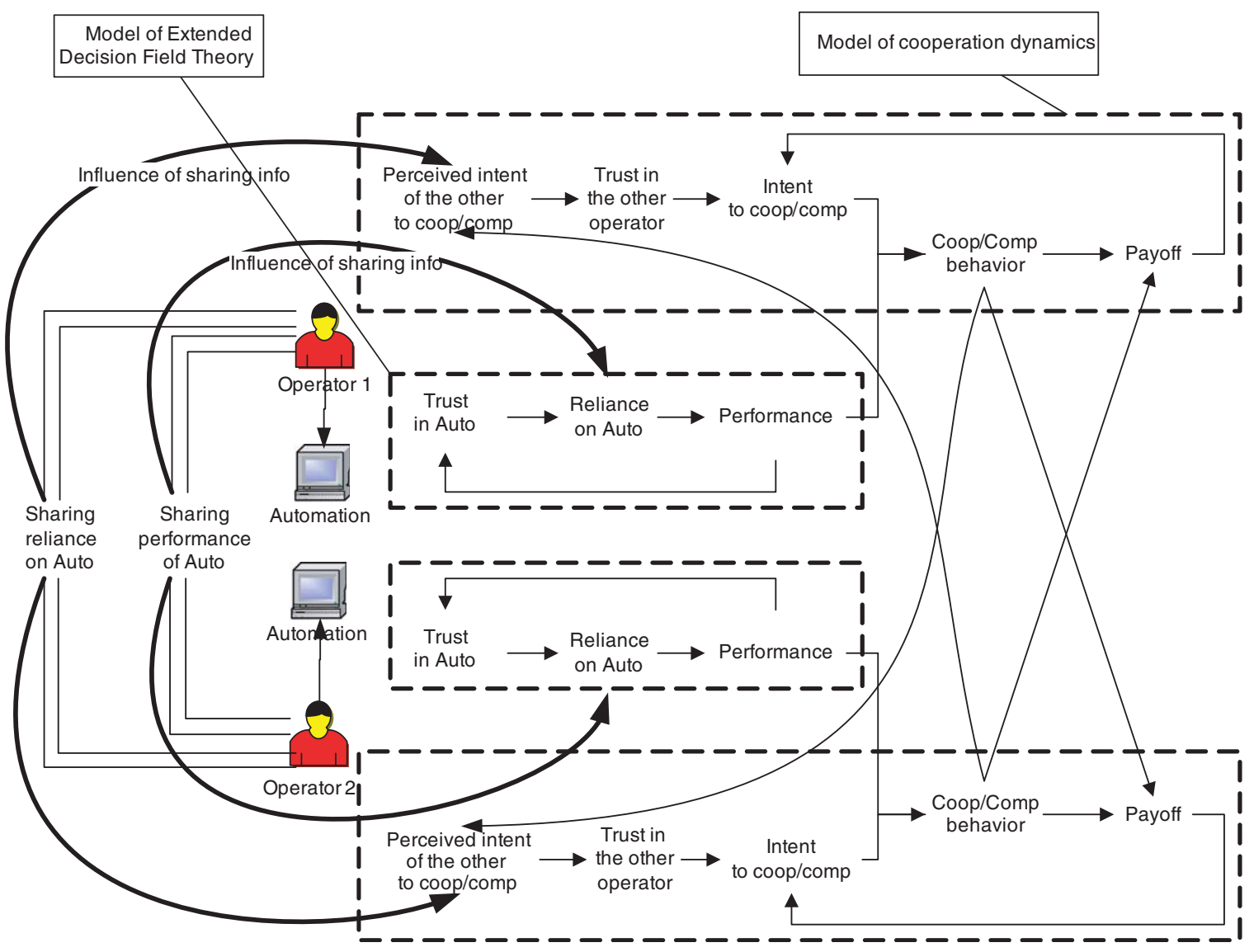

Fig. 3. Integrated model of a multi-operator multi-automation system.

deviation depends on many factors, in addition to intention, such as the accuracy of demand forecasts. Such accuracy depends in turn on the appropriateness of the operators' reliance on the forecasting system. That is, the actual inventory level might not reflect their intent if they rely on the automation inappropriately. For example, one player chooses to cooperate and sets his target inventory be $50 \%$ of the demand. His forecast of the total demand is 150 units, leading to a production of 75 units.
The actual demand turns out to be 100 units. As a result, this player's actual inventory level turns out to be $75 \%$ of the total demand, which appears to be competitive behavior.

In the model, the actual inventory level is determined by the target inventory level and the capability associated with the selected control mode (A or M). Specifically, the actual inventory $\left(I n v_{\text {Actual }}\right)$ is calculated by adding a noise term $\left(\varepsilon_{\text {Inv }}\right)$ to the target inventory (Inv $\left.v_{\text {Target }}\right)$ where the standard 
deviation of the noise term $\left(\sigma_{\text {Inv }}\right)$ is inversely proportional to the capability being used $\left(C P_{\text {In use }}\right)$ :

$$
\begin{aligned}
I n v_{\text {Actual }} & =I n v_{\text {Target }}+\varepsilon_{\text {Inv }}, \varepsilon_{\text {Inv }} \sim\left(0, \sigma_{\text {Inv }}^{2}\right), \\
\sigma_{\text {Inv }} & =\beta\left(1-C P_{\text {In use }}\right),
\end{aligned}
$$

where $C P_{\text {In use }}\left(0 \leqslant C P_{\text {In use }}<1\right)$ is derived based on profiles of $C P_{\mathrm{A}}$ and $C P_{\mathrm{M}}$ and the chosen control mode predicted by the EDFT model. The factor $\beta$ is set to define the scale of noise.

Like the EDFT model, the model of cooperation also works in a closed-loop fashion. Specifically, one operator's intention or decision to cooperate or to compete is influenced by his/her previous performance in terms of payoff. Their decision then influences their behavior and payoff for the next period. However, two important mechanisms govern cooperation. First, the appearance of cooperation/competition behavior is influenced by the forecasting performance associated with the reliance on automation, in addition to the actual intention to cooperate or compete. Second, one operator's intent to cooperate or to compete is also determined by his/her trust in the other operator, which is influenced in part by his/her perceived intent of the other, in addition to the payoff realized from the previous period.

The two mechanisms that govern cooperation provide opportunities to promote cooperation by sharing information. Two types of automation-related informationperformance of automation and reliance on automation - can be shared to enhance cooperation. In the use of forecasting systems, the operator can choose one forecasting model from different types of models and can set parameters as well as factors to generate a forecast. Then the operator has to decide whether to actually rely on the particular forecast suggested by the chosen model. That is, even when a particular model is chosen, the suggested forecast may not be relied on if the operator does not agree with it. Provided with the same forecasting system, the operators at two manufacturers might choose different forecasting models and then rely on the chosen model differently.

It is hypothesized that sharing the performance of the automation will improve operators' reliance on it and therefore make their behavior reflect their intention to cooperate/compete more precisely. The performance of automation is the degree to which the forecast of the automation matches the actual demand. Sharing such information will allow the operator to gather more information regarding the performance of different forecasting models and help calibrate the operator's trust in the forecasting models. The improved accuracy in forecasting will reduce unintentional competitive behavior (e.g., excess inventory due to an inaccurate demand forecast).

It is also hypothesized that sharing information about reliance on automation will lead to a more precise interpretation of the other's intent to cooperate or compete and therefore prevent competitive behavior induced by misinterpretation. The reliance on automation represents the degree to which the operator actually uses the forecast suggested by the selected model to generate the final forecast. This shared information will help the operator to infer the other's intent - competitive behavior initiated by the automation may not reflect competitive intent on the part of the operator. For this reason, information regarding reliance on automation may lead to a more charitable interpretation of the intent of the other when a competitive behavior is observed. For example, knowing that the other operator over-relied on the forecast suggested by a particular model (higher demand forecast as a consequence) may prevent an automatic interpretation of the excess inventory as indicating an intent to compete. A more favorable interpretation of the other's intent may enhance trust in the other and therefore promote cooperation.

Sharing these two types of information can be implemented independently. That is, the performance of the automation can be shared without information regarding the other operator's reliance on the automation. Similarly, sharing information about the reliance on automation can be achieved without providing information regarding the performance of automation.

\section{Simulation experiments}

Two simulation experiments were conducted with the combined EDFT and cooperation model to investigate how inappropriate reliance and sharing automation-related information influence cooperation. Experiment 1 directly evaluates the effect of inappropriate reliance on cooperation with the hypothesis that inappropriate reliance will trigger competitive behavior. Experiment 2 examines the effects of different types of information sharing on cooperation with three hypotheses: (1) sharing information regarding the performance of the automation will promote more appropriate reliance and induce a greater degree of cooperation; (2) sharing information regarding the other operator's reliance on automation will promote cooperation; and (3) sharing information regarding both the performance of and reliance on the automation will interact to increase cooperation.

\subsection{Model settings}

For the EDFT model, the inputs are the profiles of $C P_{\mathrm{A}}$ and $C P_{\mathrm{M}}$ and the output is the reliance on automation of an individual operator. The output from the EDFT model then becomes the input for the cooperation model. Specifically, the reliance on automation determines the actual target level and the payoffs, based on Eqs. (5)-(7) in the model of cooperation. This model then predicts the operator's decision to cooperate or compete as the output.

Each simulation run predicts a pair of operator's reliance on automation and their cooperation with each other for each of 50 sequential trials. Different simulation runs use 
different sets of random numbers for the noise terms in Eqs. (2) and (3) to represent different pairs of simulated operators. A total of 1000 pairs are used. The output, the probability of cooperation that indicates the level of cooperation for an operator at each trial, is calculated as the percentage of cooperating operators out of the 1000 simulated operators during that trial. In terms of simulation, a probability of cooperation of $80 \%$ at trial $n$ means that $80 \%$ of 1000 simulation runs predict the operator will cooperate and $20 \%$ of the runs predict the operator will compete. The operators start with an equal probability for either cooperation or competition.

The profiles of $C P_{\mathrm{A}}$ and $C P_{\mathrm{M}}$ are pre-defined. The solid and dashed lines on the top of Fig. 4 demonstrate the scaled profiles of $C P_{\mathrm{A}}$ and $C P_{\mathrm{M}}$, where the drop in automation capability characterizes the occurrence of faults. A scale of 0 to 1 is used to describe the capability level, with 0 representing minimum capability and 1 representing maximum capability. The manual capability $\left(C P_{\mathrm{M}}=0.8\right)$ remains the same and the automation capability $\left(C P_{\mathrm{A}}\right)$ varies over trials. The periodically declining capability of automation $\left(C P_{\mathrm{A}}=0.4\right)$ is used to represent automation faults. These values of $C P_{\mathrm{A}}$ and $C P_{\mathrm{M}}$ are chosen to represent a reasonable situation in which automation outperforms manual control when functioning normally, but is inferior to manual control when automation faults occur. It is assumed that automation capability $\left(C P_{\mathrm{A}}\right)$ is available to the operators only when automation is used and that manual control capability $\left(C P_{\mathrm{M}}\right)$ is always available.

In determining the actual inventory, $\beta=50$ is used in Eq. (8). Consequently, the actual inventory equals the target inventory when automation used is under normal condition $\left(C P_{\text {In use }}=1\right)$. The actual inventory fluctuates around the target with a standard deviation of 30 units when automation used is experiencing faulty condition

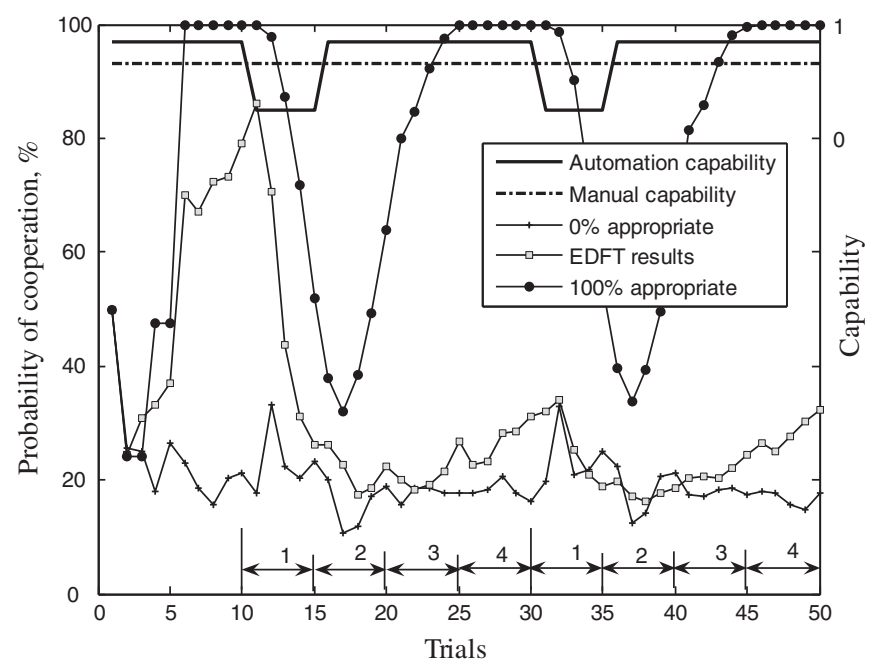

Fig. 4. Cooperation dynamics with perfect and imperfect use of automation. Note: 1: fault; 2: near post-fault normal; 3: middle post-fault normal; and 4: far post-fault normal.
$\left(C P_{\text {Inuse }}=0.4\right)$. The actual inventory fluctuates around the target with a standard deviation of 10 units when manual control is used $\left(C P_{\text {In use }}=0.8\right)$. In both experiments, the cooperation results are shown for only one operator in a pair because the two operators have quite similar results due to the symmetry of the model.

\subsection{Experiment 1: effect of inappropriate use of automation on cooperation}

\subsubsection{Experimental design}

Experiment 1 uses a mixed between and within-subjects design. The between-subject variable has three levels defined by the appropriateness of the reliance on automation. The level of appropriateness of reliance is calculated by the percentage of time the operator relies on the control mode (A or $\mathrm{M}$ ) with the higher capability. The three conditions defined by levels of appropriateness of reliance are: $0 \%$ appropriate (i.e., the control mode with a lower capability is relied on in every trial), the appropriateness associated with the reliance predicted by the EDFT model, and $100 \%$ appropriate (i.e., the control mode with a higher capability is relied on in every trial). The reliability profile represents the within-subject variable and consists of four levels of situation: during fault and then three blocks of trials increasingly removed from the fault. The second within-subject variable defines the first and second occurrence of the fault. The primary dependent variable is the simulated operator's cooperation decision (1 to cooperate or 0 to compete) in each of 50 trials. There is no shared information in Experiment 1.

The 50 trials are divided into 10 blocks with each block being 5 trials long. Four situations are particularly of interest because of their timing relative to the occurrence of automation faults: fault situations (blocks 3 and 7), near post-fault normal situations (blocks 4 and 8), middle postfault normal situations (blocks 5 and 9), and far post-fault normal situations (blocks 6 and 10). The pattern reflected in blocks 3-6 is repeated in blocks 7-10. The data were aggregated for each block to transfer the binary values of the cooperation variable to continuous values. The data in blocks 3-10 were analyzed to focus on the fault and postfault normal situations.

\subsubsection{Simulation results}

A 3 (appropriateness of reliance) $\times 4$ (situations) $\times 2$ (repetition) repeated-measures analysis of variance (ANOVA) was performed. The cooperation probabilities for the groups with $0 \%$ appropriate reliance, reliance by EDFT, and $100 \%$ appropriate reliance are $18.9 \%, 26.9 \%$, and $79.6 \%, F(2,2997)=9857.73, p<0.0001$. The cooperation probabilities in the fault, near post-fault, middle post-fault, and far post-fault normal situations are $48.3 \%, 27.1 \%$, $43.5 \%$, and $48.3 \%, F(3,8991)=1120.23, p<0.0001$. Cooperation increases as the time since the fault increases. The increased cooperation in the post-fault situations reflects the reinforcement learning mechanism of the 
cooperation model. The cooperation probability in the near post-fault normal situation is particularly low compared to other situations. This is because the operator's trust and reliance on automation has inertia and the effect of the inappropriate reliance associated with the automation faults on cooperation is delayed. The cooperation probabilities in blocks $3-6$ and in blocks $7-10$ are $42.7 \%$ and $40.9 \%, F(1,2997)=36.26, p<0.0001$. The slightly greater cooperation in blocks $3-6$ might be due to the fact that the cooperation probability for the case of reliance predicted by EDFT is still relatively high during the fault situation in blocks 3-6 because of the inertia of the reliance, as seen in Fig. 4.

The interaction between the appropriateness of reliance and situations is significant, $F(6,8991)=687.97$, $p<0.0001$. The cooperation drops more dramatically after the fault occurs but recovers to $100 \%$ cooperation sooner with $100 \%$ appropriate reliance compared to with less appropriate reliance. The case of $100 \%$ appropriate reliance minimizes the effect of inappropriate reliance on cooperation. The interaction between the appropriateness of reliance and repetition is significant, $F(2,2997)=66.77$, $p<0.0001$. The greater cooperation seen in blocks $3-6$ than in blocks $7-10$ is particularly significant for the reliance predicted by EDFT, in which the cooperation is close to $100 \%$ before the first automation fault and does not recover to the same high level afterwards within the postfault normal situations. The interaction between the situation and repetition is significant, $F(3,8991)=44.03$, $p<0.0001$. The greater cooperation in blocks $3-6$ than in blocks $7-10$ is only observed for the fault situation.

Fig. 4 shows the profiles of cooperation for the three levels of appropriateness. In the Pre-fault normal situations (blocks 1 and 2), operators with 100\% appropriate reliance quickly learn to cooperate. That is, a pair of operators quickly reaches mutual cooperation (they have similar cooperation profiles). In contrast, when the appropriateness is less than $100 \%$, mutual cooperation is less likely. In the worst case, with $0 \%$ appropriate reliance, the probability of cooperation quickly drops and fluctuates around $20 \%$ thereafter.

\subsubsection{Discussion}

Cooperation drops dramatically after the automation fails. This drop in cooperation can be explained in the context of the game scenario described previously. The operators' desired contribution to the actual demand depends on their forecasted demand. If the operator's desired contribution is $50 \%$, reflecting an intention to cooperate, and the forecasted demand is higher than actual demand, the contribution will be greater than $50 \%$, signifying unintentionally competitive behavior. Operators with $100 \%$ appropriate use of automation and their forecasts more precisely match the actual demand. Those with $0 \%$ appropriateness choose the less capable control mode option, leading to less precise forecasts and greater competitive behavior. Operators with appropriateness predicted by the EDFT model also compete because they sometimes choose the more capable control mode and sometimes the less capable control mode option. The cooperation of operators with $100 \%$ appropriate reliance recovers relatively quickly after the faults pass, and operators with reliance predicted by the EDFT model are much less likely to recover.

\subsection{Experiment 2: influence of sharing automation-related information on cooperation}

\subsubsection{Experimental design}

Experiment 2 uses a mixed between and within subject design. The within-subject conditions define the profile of the automation failure and are the same as those in Experiment 1. Two between-subject factors define the four between-subject conditions: sharing information of automation performance and sharing reliance on automation. Table 3 lists the four conditions and describes how they were implemented within the model. When two operators share information regarding automation performance, $C P_{\mathrm{A}}(n-1)$ will be available to update the $B_{\mathrm{A}}(n)$ of one operator (see Eq. (1)) when the other operator is using automation. When sharing information about reliance on automation, the original levels of $\theta_{C / D}^{+}(=+0.2)$ and $\theta_{C / D}^{-}$ $(=-0.2)$ will be lowered by $25 \%$ (i.e., 0.05$)$. Specifically, $\theta_{C / D}^{+}=+0.15$ and $\theta_{C / D}^{-}=-0.25$ replace $\theta_{C / D}^{+}=+0.2$ and $\theta_{C / D}^{-}=-0.2$. Because the shared information regarding the other's reliance will most likely make a difference when the other's reliance is not appropriate, the thresholds will be lowered only during the trials when the other operator uses automation inappropriately. A lower level of $\theta_{C / D}^{+}$or $\theta_{C / D}^{-}$ corresponds to the situation where people are more willing to cooperate when compared to a higher level of $\theta_{C / D}^{+}$or $\theta_{C / D}^{-}$. Therefore, such a setting is used to characterize the situation where the operator generates a more charitable interpretation of the other's intent to cooperate or compete. The reliance on automation predicted from EDFT is used in Experiment 2.

\subsubsection{Simulation results}

A 2 (sharing automation performance) $\times 2$ (sharing reliance on automation) $\times 4$ (situations) $\times 2$ (repetition) repeated-measures ANOVA was performed. The main and two-way interaction effects of four situations and

Table 3

Experiment 2: Four conditions defined by two factors

\begin{tabular}{llll}
\hline & \multicolumn{2}{l}{ Reliance on automation } \\
\cline { 3 - 4 } & & Not sharing & Sharing \\
\hline Automation performance & Not sharing & 1 & 3 \\
& Sharing & 2 & 4 \\
\hline
\end{tabular}

Sharing automation performance is set by EDFT model, $\mathrm{CP}_{\mathrm{A}}(n-1)$ is available; sharing reliance on automation is set by Cooperation model, lower $\theta_{C / D}^{+}$and $\theta_{C / D}^{-}$by $25 \%$. 
repetition on cooperation are similar to those in Experiment 1 and will not be discussed. The main effect of sharing each type of information on cooperation and the associated interaction effects are reported.

The cooperation probabilities are $33.3 \%$ and $37.1 \%$ without and with sharing of automation performance information, $F(1,3996)=57.43, p<0.0001$. The interaction between the sharing of automation performance and the four situations is significant, $F(1,12000)=18.92$, $p<0.0001$. The cooperation probabilities for the four situations are $44.5 \%, 26.5 \%, 28.0 \%$, and $34.1 \%$ for the sharing automation performance condition and are $48.3 \%$, $27.2 \%, 30.9 \%$, and $41.9 \%$ for the not-sharing condition. Sharing automation performance improves the cooperation more in the middle and most in the far post-fault compared to the near post-fault normal situations. The interaction between sharing performance and repetition is significant, $F(1,3996)=7.93, p=0.0049$. The improvement in cooperation is less in blocks 3-6 than in blocks 7-10. Fig. 5 shows the effect of sharing automation performance on cooperation. The error bars depict a $95 \%$ confidence interval of the mean of binary values of cooperation. The effect of improvement is more evident in the post-fault normal situations as shown in Fig. 5. The cooperation recovers more rapidly when the information is shared in post-fault normal situations.

The cooperation probabilities are $29.1 \%$ and $41.3 \%$ without and with sharing information on reliance on automation, $F(1,3996)=598.38, p<0.0001$. The interaction between sharing reliance and the four situations is significant, $F(1,12000)=4.41, p=0.0042$. The cooperation probabilities for the four situations are $41.2 \%, 20.1 \%$, $22.9 \%$, and $32.2 \%$ for the sharing reliance condition and $51.6 \%, 33.7 \%, 36.0 \%$, and $43.9 \%$ for the not-sharing condition. Sharing performance improves cooperation most in the near post-fault normal situations. The interaction between sharing performance and repetition is not significant. Fig. 6 compares the cooperation profiles with sharing only reliance on automation to those without sharing any information. The effect of the shared information is more significant when the operators use automation less appropriately. A greater level of improvement in cooperation is observed in post-fault normal situations. The automation faults lead to inappropriate reliance on automation, which can cause more competition.

The interaction between sharing performance and sharing reliance is not significant, $F(1,3996)=1.69$, $p=0.19$. Fig. 7 shows that sharing both types of information simply increases the level of cooperation more

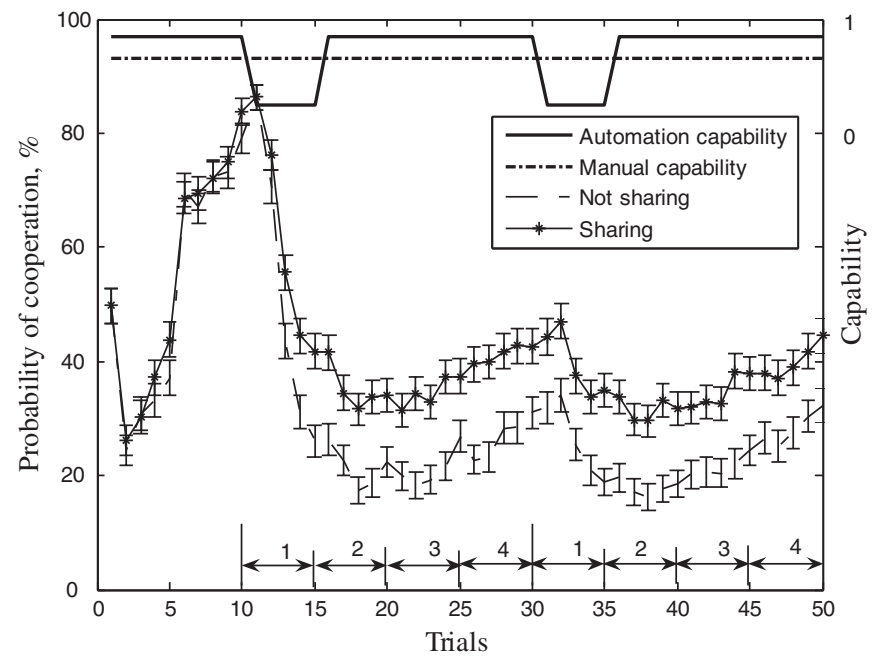

Fig. 6. The influence of sharing the reliance on automation on cooperation. Note: 1: fault; 2: near post-fault normal; 3: middle postfault normal; and 4: far post-fault normal.

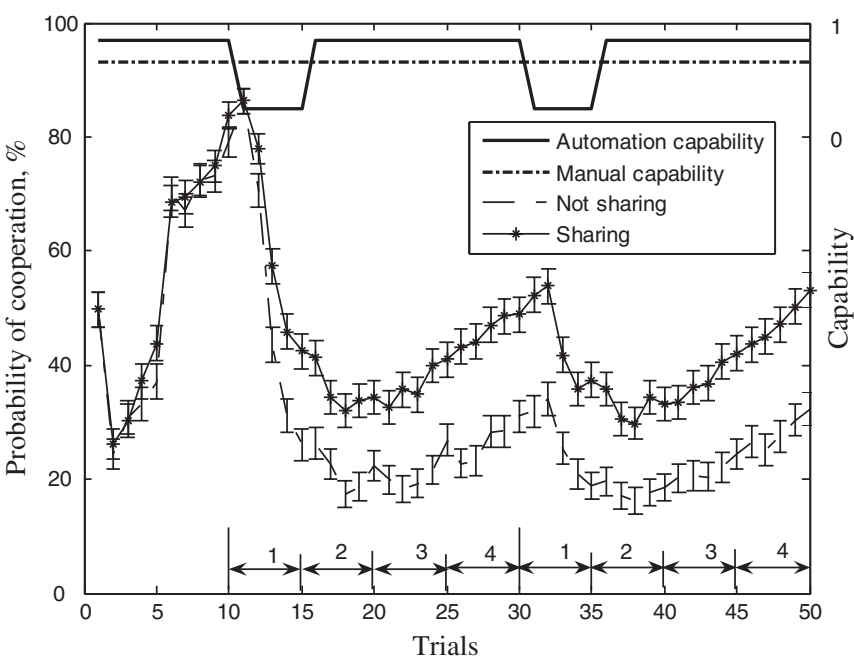

Fig. 7. The influence of sharing both automation performance and reliance on cooperation. Note: 1 : fault; 2 : near post-fault normal; 3: middle post-fault normal; and 4: far post-fault normal.
Fig. 5. The influence of sharing automation performance on cooperation Note: 1: fault; 2: near post-fault normal; 3: middle post-fault normal; and 4: far post-fault normal. 
than sharing either alone. The model shows that sharing these two types of information influences cooperation in an additive manner.

Fig. 8 shows the improvement in cooperation when sharing information (performance, reliance, or both) compared to not sharing any information trial by trial. It demonstrates that the effect of sharing each type of information on cooperation varies over different situations. Sharing automation performance has relatively less effect on cooperation in the fault and near post-fault situations and starts to improve the cooperation more in the middle and far post-fault situations. This is due to the fact that sharing performance improves cooperation by improving operators' appropriate reliance on automation and the inertia of reliance delays the effect of sharing performance on cooperation until the middle and far post-fault situations. Sharing reliance improves cooperation by the greatest percentage in the near post-fault situation. The reason why the relative improvement is less in fault situations is that the inappropriate reliance on automation associated with the automation fault has such a significant impact on cooperation right after the fault occurs so that even sharing information has relatively little effect on cooperation. An explanation for the relatively smaller effect on cooperation in the middle and far post-fault situations is that the learning effect (i.e., operators tend to cooperate more over time) is less significant when the cooperation probability becomes greater.

\subsubsection{Discussion}

The improved cooperation between operators that results from sharing automation performance can be explained by the effect of sharing such information on the operator's reliance on automation. Fig. 9 shows the time-varying distribution of operators' reliance on auto-

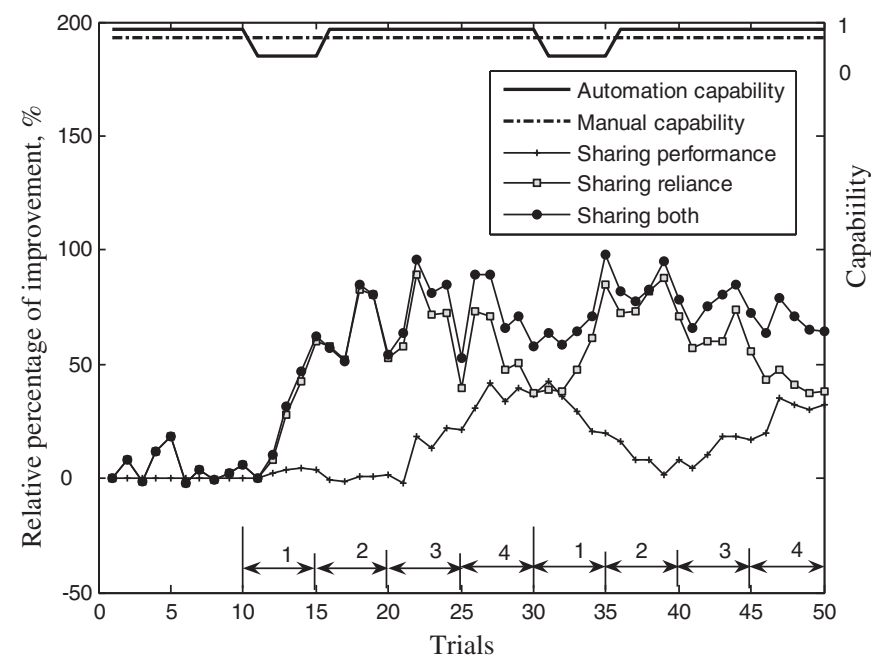

Fig. 8. Comparison of relative percentages of improvement of cooperation. Note: 1: fault; 2: near post-fault normal; 3: middle post-fault normal; and 4: far post-fault normal.

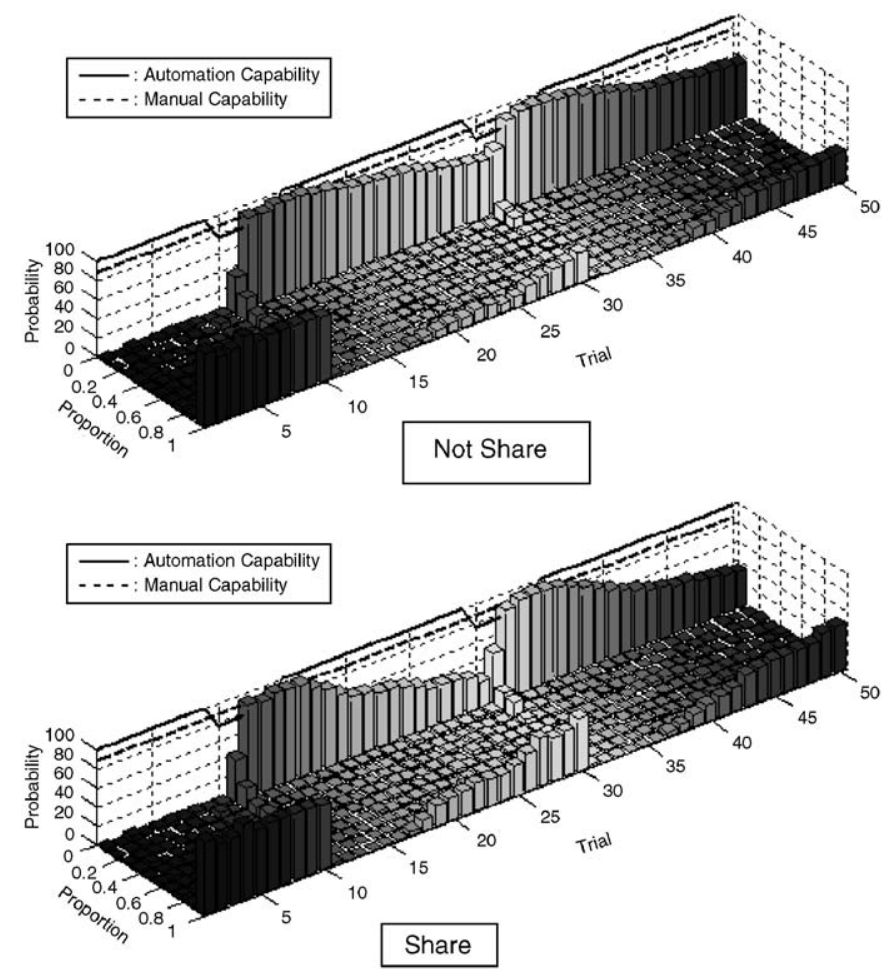

Fig. 9. Distribution of reliance.

mation predicted by the EDFT model. The proportion of reliance, with a scale of $0-1$, is evenly divided into 10 bins to signify 10 levels of reliance. Each level represents the proportion of time spent in automatic control during each trial (e.g., 0.2 represents $20 \%$ of time spent in automatic control). The probability, with a scale of $0-100$, is calculated from the percentage of simulated operators who adopted each of the 10 levels of reliance for each trial. Similar to Fig. 4, Fig. 9 demonstrates the scaled profiles of $C P_{\mathrm{A}}$ and $C P_{\mathrm{M}}$ on the vertical surface. Fig. 9 shows that when sharing automation performance, more operators return to automatic control when the automation returns to normal during trials $16-30$ and $36-50$. That is, sharing automation performance information leads operators to rely on the automation more appropriately. However, when automation returns to normal and outperforms manual control, the operators are more likely to keep using manual control because of the inertia of their trust in automation and self-confidence. Reliance is more appropriate because the shared information enables operators to access the automation capability even when the automation is not used. A more appropriate reliance then leads to a greater level of cooperation, which is already illustrated by Experiment 1 .

Sharing reliance on automation improves cooperation substantially. Fig. 6 illustrates the improvement when lowering the thresholds $\left(\theta_{C / D}^{+}\right.$and $\left.\theta_{C / D}^{-}\right)$by $25 \%$. The simulation experiments show that when the thresholds are lowered by more than $10 \%$, cooperation improves 
significantly. Additionally, the more the thresholds are reduced, the greater the improvement in cooperation.

The reasons why sharing automation performance or reliance improves cooperation can be interpreted in the context of the game scenario described previously. Sharing automation performance improves the operator's reliance on automation and the more appropriate reliance moderates the mismatch between the operator's intent and the actual behavior. The reduced mismatch promotes cooperation by avoiding unintended competitive behaviors caused by inappropriate use of automation. Sharing reliance on automation generally increases cooperation by leading to a greater level of willingness to cooperate even when observing the competitive behavior of the other. Specifically, the information about the other operator's reliance on automation together with the operator's own experience with automation performance may explain the other operator's competitive behavior when it is caused by inappropriate use of automation. Sharing the other operator's reliance on automation leads to a more charitable interpretation of the other's intent and therefore increases trust. The consequence of enhanced trust is an increased chance of cooperation. These interpretations need to be further validated with experiments that include human participants. Sharing performance-related information influences the judgments of previous performance and sharing reliance information influences trust in the other. That is, these two types of information sharing influence cooperation independently, which explains the lack of interaction observed in the simulation results. Controlled experiments with human subjects will test the hypotheses as well as validate and refine the model.

\section{Conclusions}

We examined how reliance on automation influences cooperation and how sharing two types of automationrelated information mediates this influence in the context of a two-manufacturer one-retailer SC system. Simulation results show that inappropriate reliance hinders cooperation, and that sharing automation performance information promotes more appropriate reliance and as a result more cooperation among operators. Sharing reliance information promotes cooperation. Sharing both performance and reliance information enhances cooperation in an additive manner. Overall cooperation depends on the appropriate use of automation and sharing automationrelated information can have a profound effect on cooperation.

This is the first study to address how interaction among multiple operators and multiple automation influences cooperation in a SC system. This study demonstrates that cooperation can be promoted by sharing automationrelated information to avoid unintentional competitive behavior as well as to lead to a more charitable interpretation of the other's intent.

\section{Acknowledgements}

We would like to thank the editor and two anonymous reviewers as well as the colleagues from the Cognitive Systems Lab for their helpful comments and suggestions on the revision of the paper.

\section{References}

Andreoni, J., Miller, J.H., 1993. Rational cooperation in the finitely repeated prisoners-dilemma - experimental-evidence. Economic Journal 103 (418), 570-585.

Anupindi, R., Bassok, Y., Zemel, E., 2001. A general framework for the study of decentralized distribution systems. Manufacturing and Service Operations Management 3 (4), 349-368.

Barratt, M., 2004. Understanding the meaning of collaboration in the supply chain. Supply Chain Management - an International Journal 9 (1), 30-42.

Burgers, W.P., Hill, C.W.L., Kim, W.C., 1993. A theory of global strategic alliances - the case of the global auto industry. Strategic Management Journal 14 (6), 419-432.

Busemeyer, J.R., Townsend, J.T., 1993. Decision field theory: a dynamic cognitive approach to decision making in an uncertain environment. Psychological Review 100 (3), 432-459.

Cachon, G., Netessine, S., 2004. Game theory in supply chain analysis. In: Simchi-Levi, D., Wu, D., Shen, Z.-J. (Eds.), Handbook of quantitative supply chain analysis: Modeling in the eBusiness era. Kluwer.

Cachon, G.P., 2002. Supply chain coordination with contracts. In: Graves, S., Kok, T. (Eds.), Handbooks in Operations Research and Management Science: Supply Chain Management. North-Holland, Amsterdam.

Cachon, G.P., Zipkin, P.H., 1999. Competitive and cooperative inventory policies in a two-stage supply chain. Management Science 45 (7), 936-953.

Colman, A.M., 1982. Game Theory and Experimental Games: The Study of Strategic Interaction. Pergamon Press, New York.

Colman, A.M., 1995. Game Theory and its Applications in the Social and Biological Sciences. Butterworth-Heinemann, London.

Colman, A.M., 2003. Cooperation, psychological game theory, and limitations of rationality in social interaction. Behavioral and Brain Sciences 26 (2), 139.

Cooper, R., DeJong, D.V., Forsythe, R., Ross, T.W., 1996. Cooperation without reputation: experimental evidence from prisoner's dilemma games. Games and Economic Behavior 12 (2), 187-218.

Davis, M.D., 1983. Game Theory: A Nontechnical Introduction. Basic Books Inc., New York.

Dawkins, R., 1988. The Selfish Gene. Oxford University Press, Oxford.

Dugatkin, L.A., Mestertongibbons, M., Houston, A.I., 1992. Beyond the prisoners-dilemma - toward models to discriminate among mechanisms of cooperation in nature. Trends in Ecology and Evolution 7 (6), 202-205.

Dussauge, P., Garrette, B., Mitchell, W., 2004. Asymmetric performance: the market share impact of scale and link alliances in the global auto industry. Strategic Management Journal 25 (7), 701-711.

Elahi, E., Benjaafar, S., Donohue, K., 2003. Inventory competition in make-to-stock systems. In: Proceedings of the Aegean International Conference on Analysis of Manufacturing Systems (4th Meeting).

Foddy, M., Smithson, M., Schneider, S., Hogg, M., 1999. Resolving Social Dilemmas: Dynamic, Structural, and Intergroup Aspects. Psychology Press.

Gao, J., Lee, J.D. Extending the decision field theory to model operators' reliance on automation in supervisory control situations. IEEE Transactions on Systems, Man, and Cybernetics, in press.

Gavirneni, S., Kapuscinski, R., Tayur, S., 1999. Value of information in capacitated supply chains. Management Science 45 (1), 16-24. 
Gutnisky, D.A., Zanutto, B.S., 2004. Cooperation in the iterated prisoner's dilemma is learned by operant conditioning mechanisms. Artificial Life 10 (4), 433-461.

Huang, G.Q., Lau, J.S.K., Mak, K.L., 2003. The impacts of sharing production information on supply chain dynamics: a review of the literature. International Journal of Production Research 41 (7), $1483-1517$.

Kumar, K., vanDissel, H.G., 1996. Sustainable collaboration: managing conflict and cooperation in interorganizational systems. Mis Quarterly 20 (3), 279-300.

Ledyard, J.O., 1995. Public goods: a survey of experimental research. In: Kagel, J.H., Roth, A.E. (Eds.), Handbook of Experimental Economics. Princeton University Press, Princeton, NJ.

Lee, H.L., Billington, C., 1992. Managing supply chain inventory-pitfalls and opportunities. Sloan Management Review 33 (3), 65-73.

Lee, H.L., Whang, S.J., 2000. Information sharing in a supply chain. International Journal of Technology Management 20 (3-4), 373-387.

Lee, J.D., See, K.A., 2004. Trust in technology: designing for appropriate reliance. Human Factors 46 (1), 50-80.

Lew, S.E., Wedemeyer, C., Zanutto, B.S., 2001. Role of unconditioned stimulus prediction in the operant learning: a neural network model. In: Proceedings of the IEEE Conference on Neural Networks.

Li, L.D., 2002. Information sharing in a supply chain with horizontal competition. Management Science 48 (9), 1196-1212.

Lippman, S.A., McCardle, K.F., 1997. The competitive newsboy. Operations Research 45 (1), 54-65.

Lipton, E., Pena, R.P., Wald, M.L., 2003. Overseers missed big picture as failures led to blackout. New York Times, 9/13/03.

Luce, R.D., Raiffa, H., 1957. Games and Decisions: Introduction and Critical Survey. Wiley, New York.

Macy, M.W., 1991. Learning to cooperate-stochastic and tacit collusion in social-exchange. American Journal of Sociology 97 (3), 808-843.

Macy, M.W., Flache, A., 2002. Learning dynamics in social dilemmas. Proceedings of the National Academy of Sciences of the United States of America 99, 7229-7236.

Mahajan, S., van Ryzin, G., 2001. Inventory competition under dynamic consumer choice. Operations Research 49 (5), 646-657.

Mishra, B.K., Raghunathan, S., 2004. Retailer- vs. vendor-managed inventory and brand competition. Management Science 50 (4), 445-457.

Napier, R., 2001. Global trends: cooperation, learning a lesson from the global giants. Retrieved 11/2, 2004, from http://farmcentre.com/ english/cfm/oct-nov01/page4.htm

Narasimhan, R., Mahapatra, S., 2004. Decision models in global supply chain management. Industrial Marketing Management 33 (1), 21-27.

Nash, J.F., 1950. Equilibrium points in n-person games. Proceedings of the National Academy of Sciences 36, 48-49.

Parasuraman, R., Riley, V., 1997. Humans and automation: use, misuse, disuse, abuse. Human Factors 39 (2), 230-253.
Parasuraman, R., Sheridan, T.B., Wickens, C.D., 2000. A model for types and levels of human interaction with automation. IEEE Transactions on Systems Man and Cybernetics-Part A: Systems and Humans 30 (3), 286-297.

Parlar, M., 1988. Game theoretic analysis of the substitutable product inventory problem with random demands. Naval Research Logistics 35 (3), 397-409.

Raghunathan, S., 2003. Impact of demand correlation on the value of and incentives for information sharing in a supply chain. European Journal of Operational Research 146 (3), 634-649.

Rudi, N., Netessine, S., 1999. Demand substitution in retail: centralization and competition. Unpublished manuscript, Working Paper, Simon School of Business Administration, University of Rochester, Rochester, NY.

Sabath, R., Fontanella, J., 2002. The unfulfilled promise of supply chain collaboration. Supply Chain Management Review (July/August), 24-29.

Sahin, F., Robinson, E.P., 2002. Flow coordination and information sharing in supply chains: review, implications, and directions for future research. Decision Sciences 33 (4), 505-536.

Selten, R., Stoecker, R., 1986. End behavior in sequences of finite prisoners-dilemma supergames - a learning-theory approach. Journal of Economic Behavior and Organization 7 (1), 47-70.

Sigmund, K., 1993. Games of Life. Oxford University Press, Oxford.

Simon, H., 1992. Decision making and problem solving. In: Zey, M. (Ed.), Decision Making: Alternatives to Rational Choice Models. Sage, Newbury Park, CA, pp. 32-53.

Smith, J.M., 1982. Evolution and the Theory of Games. Cambridge University Press, New York.

Stephens, D.W., McLinn, C.M., Stevens, J.R., 2002. Discounting and reciprocity in an iterated prisoner's dilemma. Science 298 (5601), 2216-2218.

Townsend, J.T., Busemeyer, J.R., 1995. Dynamic representation of decision-making. In: Port, R.F., Gelder, T.V. (Eds.), Mind as Motion. MIT Press, Cambridge, pp. 101-120.

US-Canada Power System Outage Task Force, 2003. Interim report: Causes of the August 14th blackout in the United States and Canada, Available: www.doe.gov

van Ryzin, G., Mahajan, S., 1999. Supply Chain Coordination Under Horizontal Competition. Columbia University Graduate School of Business, New York.

von Neumann, J., Morgenstern, O., 1944. Theory of Games and Economic Behavior. Princeton University Press, Princeton, NJ.

Wu, T., O'Grady, P., 2004. An extended Kalman filter for collaborative supply chains. International Journal of Production Research 42 (12), 2457-2475.

Zhang, H.T., 2002. Vertical information exchange in a supply chain with duopoly retailers. Production and Operations Management 11 (4), 531-546. 